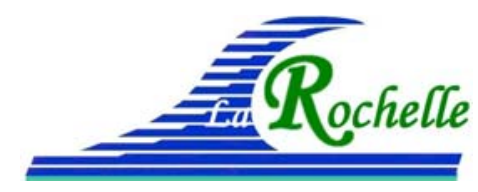

XVèmes Journées Nationales Génie Côtier - Génie Civil

La Rochelle, 29 au 31 mai 2018

DOI:10.5150/jngcgc.2018.021 ～(C) Editions Paralia CFL

disponible en ligne - http://www.paralia.fr - available online

\title{
Modélisation numérique de la dispersion des rejets par clapage des sédiments dragués dans le port de Pandop (Nouvelle Calédonie)
}

\author{
Julien BAILLS ${ }^{1}$, Jérémy DUGOR ${ }^{1}$, Didier RIHOUEY ${ }^{1}$ \\ 1. CASAGEC INGENIERIE, 18 rue Maryse Bastié, Zone de Maignon, 64600 Anglet, \\ France. \\ contact@casagec.fr
}

\section{Résumé :}

La présente étude a pour but d'évaluer si le clapage des matériaux du port de Pandop (Nouvelle-Calédonie) dans une zone d'immersion située au large du lagon est possible sans risque d'impacter le récif barrière.

La première étape de ce travail fut d'appréhender les conditions océano-climatiques pouvant impacter le site d'étude afin de définir des scénarios représentatifs de ce climat particulier notamment en raison de la concomitance de plusieurs phénomènes : la marée astronomique, les circulations océaniques à grande échelle et les états de mer.

La climatologie des états de mer a été appréhendée à partir des données de houle (sur une période de 8 ans) issues des modèles WAM. La marée astronomique est prise en compte avec le modèle global TPXO. Les courants océaniques à grande échelle autour de la Nouvelle Calédonie sont complexes et variables en temps et espace. Ces courants sont étudiés à partir des résultats des modèles tridimensionnels Mercator pour différentes profondeurs et sur une période de 10 ans. Les analyses statistiques montrent une variabilité saisonnière importante des courants mais également une forte variabilité suivant la profondeur notamment en raison de la présence d'une pycnocline marquée située à une profondeur d'environ $60 \mathrm{~m}$.

Une fois tous ces processus caractérisés, elles permettent de forcer le modèle mis en place à l'échelle locale à l'aide du code de calcul Telemac 3D. Le modèle, qui a nécessité le développement d'un module spécifique pour assimiler les circulations océaniques globales issues du modèle Mercator, couvre le quart Nord-Ouest de la Nouvelle Calédonie et compte 41200 nœuds de calculs et 10 couches.

La modélisation de la dispersion des sédiments clapés a ainsi permis de définir une zone de largage des matériaux offrant le meilleur compromis "durée du cycle de dragage / impact sur le récif barrière". Enfin, les effets cumulatifs des clapages successifs ont pu être appréhendés via des scénarios de dragage long terme.

\section{Mots-clés :}

Clapages, Nouvelle-Calédonie, Circulations océaniques, Modélisation numérique 3D. 


\section{Thème 2 - Dynamique sédimentaire}

\section{Introduction}

\subsection{Contexte général et objectifs de l'étude}

Le port de Pandop est sujet à des phénomènes de sédimentation qui nécessitent la réalisation d'opérations de dragages afin de maintenir les cotes d'exploitation.

Une des filières d'évacuation des sédiments dragués serait en mer. Il est donc nécessaire de produire des études de modélisation numérique hydrosédimentaire de détail afin de :

- Caractériser les conditions océano-climatiques du site d'étude,

- Caractériser l'évolution de la dispersion des rejets afin de définir la zone de clapage la plus proche du lagon tout en étant suffisamment éloignée pour ne pas avoir de risque d'impact sur le récif barrière,

- Appréhender l'impact du cumul des rejets.

Pour cela, un modèle numérique hydrosédimentaire tridimensionnel a été mis en place afin de représenter tous les processus impactant la zone d'étude. Deux modules spécifiques ont ainsi été développés afin de représenter les circulations océaniques globales et modéliser les clapages.

\subsection{Situation géographique}

Le port de Pandop se situe dans la Province Nord de Nouvelle-Calédonie au niveau de la pointe de Koumac. Afin de larguer les sédiments de dragage, les barges devront traverser le lagon sur environ $11,5 \mathrm{~km}$ (6,2 milles nautique) jusqu'à la passe de Koumac. Les fonds passent alors de 3 à $15 \mathrm{~m}$ dans le lagon à plus de $1000 \mathrm{~m}$ de profondeur à $3 \mathrm{~km}$ à l'extérieur de la passe.

Devant la passe de Koumac, le récif barrière représente une baie de $9 \mathrm{~km}$ de diamètre où débouchent deux passes : la passe de Koumac et la passe de Deverd.

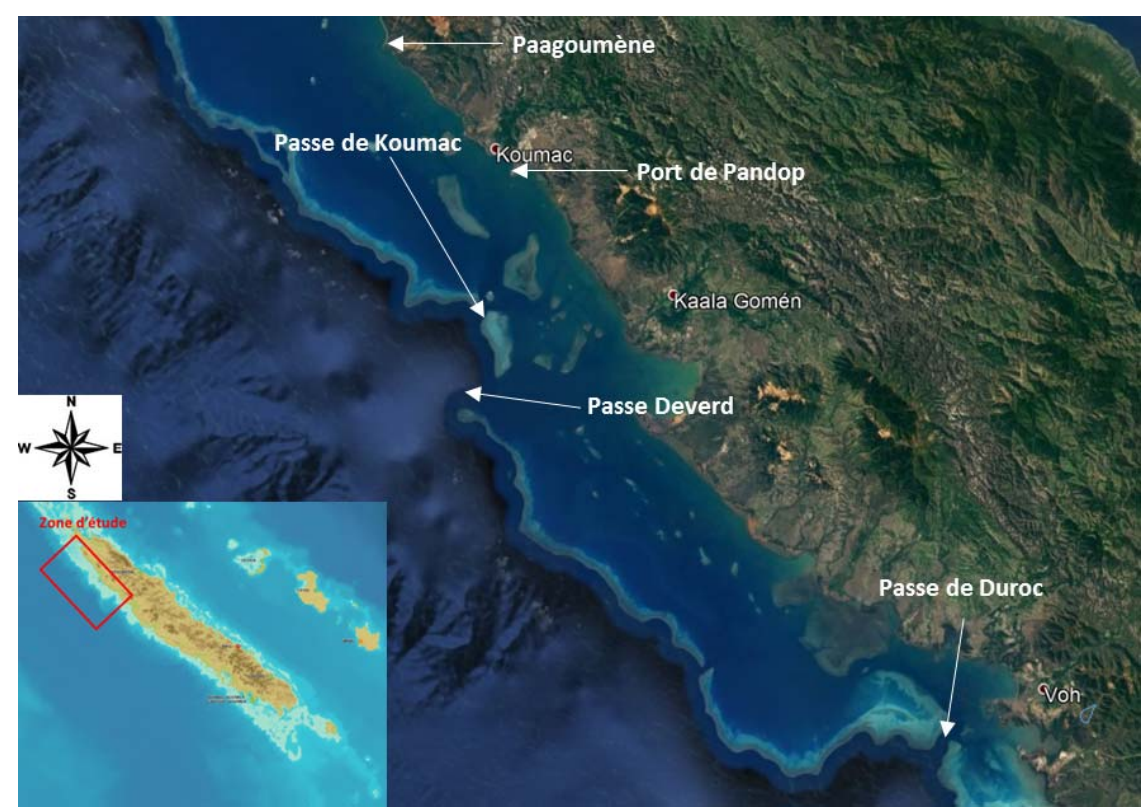

Figure 1. Localisation de la zone d'étude (Nord-Ouest de la Nouvelle Calédonie). 


\section{XVèmes Journées Nationales Génie Côtier - Génie Civil \\ La Rochelle, 29 au 31 mai 2018}

\section{Contexte océano-climatique}

\subsection{Climatologie générale}

Le climat de la Nouvelle-Calédonie est caractérisé par deux saisons principales :

- La saison des cyclones caractérisée par le passage de dépressions et de cyclones tropicaux et un régime de vents de Sud-Est à Est (régime d'alizés)

- La saison fraiche caractérisée par des vents dominants de secteur Est-Sud-Est mais aussi de passage de fronts froids accompagnés de précipitations abondantes et de vents de secteur Ouest pouvant souffler en tempête (coups d'Ouest).

La marée est de type semi-diurne à inégalité diurne et le marnage maximum est de 1,67m sur le secteur de Pandop.

\subsection{Climat de houle au large}

Les données d'états de mer issues des modèles globaux de houle WAM (KBR, 2002) ont été extraites au large de Pandop (point d'extraction localisé en longitude $164,5^{\circ} \mathrm{E}$ et latitude 21, $0^{\circ} \mathrm{S}$ ). Ces données de houle couvrent une période de 8 ans de 1994 à 2001. En synthèse, les informations à retenir de ces statistiques de houle sont les suivantes :

- Les directions principales de provenance sont du Sud-Est $\left(135^{\circ} N\right)$ au Sud-Sud-Ouest $\left(210^{\circ} \mathrm{N}\right)$ avec près de $95 \%$ des états de mer incidents.

- Les hauteurs significatives sont inférieures à $1,5 \mathrm{~m}$ pendant $23 \%$ du temps, inférieures à $2 \mathrm{~m}$ pendant $55 \%$ du temps et inférieures à $3 \mathrm{~m}$ pendant $92 \%$ du temps.

- Les états de mer inférieurs à 3,0 $\mathrm{m}$ ont généralement des périodes pics de l'ordre de 9 à 14 s. Plus rarement, certains états de mer peuvent atteindre des hauteurs significatives de plus de $6 \mathrm{~m}$ et des périodes longues pouvant atteindre $20 \mathrm{~s}$.

\subsection{Circulation grande échelle}

Les courants océaniques à grande échelle autour de la Nouvelle Calédonie sont complexes et variables en temps et espace et sont donc difficile à caractériser (NSR, 2001).

Afin d'étudier ces circulations, les résultats des modèles Mercator (BRASSEUR et al., 2005) ont été récupérés afin de créer une base de données. Cette base de données couvre une période de 10 ans (de début 2007 à fin 2016). Les courants sont, de plus, récupérés pour différentes profondeurs (surface, $100 \mathrm{~m}, 400 \mathrm{~m}$ et $1000 \mathrm{~m}$ ).

Afin de caractériser ces circulations grande échelle le long de la côte Ouest de la Nouvelle Calédonie, les courants sont étudiés pour un point situé au large de la passe de Koumac. A partir des valeurs récupérées en ce point, des roses de courants ont ainsi pu être construites pour les différentes profondeurs récupérées mais également pour les différentes saisons (saison fraîche et saison des cyclones). 


\section{Thème 2 - Dynamique sédimentaire}

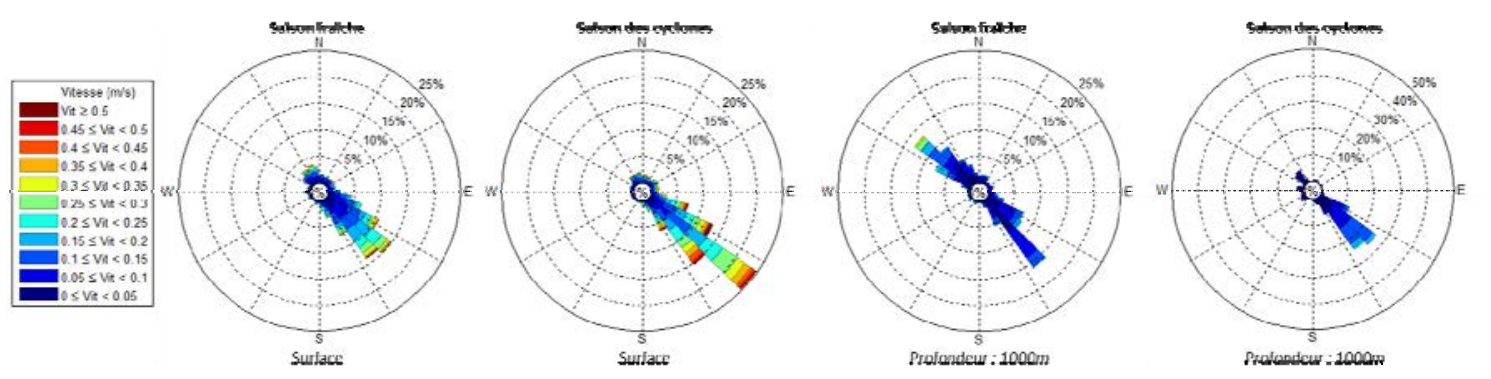

Figure 2. Rose des courants au large de Pandop issus des modèles Mercator.

Les roses de courants montrent une variabilité saisonnière importante.

En effet, pendant la saison des cyclones (de mi-Novembre à mi-Avril), les courants sont principalement dirigés vers le Sud-Est avec des vitesses moyennes de l'ordre de 0,15 à $0,5 \mathrm{~m} / \mathrm{s}$ en surface. De plus, les directions des courants sont homogènes sur toute la colonne d'eau et les vitesses sont bien plus faibles au fond.

Pendant la saison fraiche (de mi-Avril à mi-Novembre), les courants en surface sont toujours globalement dirigés vers le Sud-Est avec cette fois des vitesses plus faibles de l'ordre de 0,05 à $0,3 \mathrm{~m} / \mathrm{s}$. Par ailleurs, les directions des courants peuvent s'inverser sous la thermocline avec des courants dirigés vers le Nord-Ouest.

\section{Description du modèle numérique}

\subsection{Emprise, maillage et forçages}

Le code de calcul Telemac 3D a été utilisé afin de modéliser les écoulements tridimensionnels et le transport des matières en suspension clapées autour de la zone d'étude. Le modèle couvre le quart Nord-Ouest de la Nouvelle-Calédonie et est composé de 41200 nœuds de calculs. Les tailles de mailles varient de $1000 \mathrm{~m}$ au large à $100 \mathrm{~m}$ sur les platiers récifaux et à $50 \mathrm{~m}$ autour de la zone de clapage.

Le maillage vertical comprend 10 couches. La discrétisation verticale a été raffinée entre la surface et la pycnocline. Ce maillage a été construit afin d'obtenir un ratio précision-temps de calcul acceptable.

Les données bathymétriques utilisées pour générer le modèle numérique de terrain sont:

- Bathymétrie GEBCO (General Bathymetric Chart of the Oceans) du large,

- Bathymétrie ZONECO (Zone économique de Nouvelle-Calédonie) du proche côtier.

Les frontières et les forçages du modèle sont schématisés sur la figure $3 \mathrm{~b}$. 


\section{XVèmes Journées Nationales Génie Côtier - Génie Civil \\ La Rochelle, 29 au 31 mai 2018}
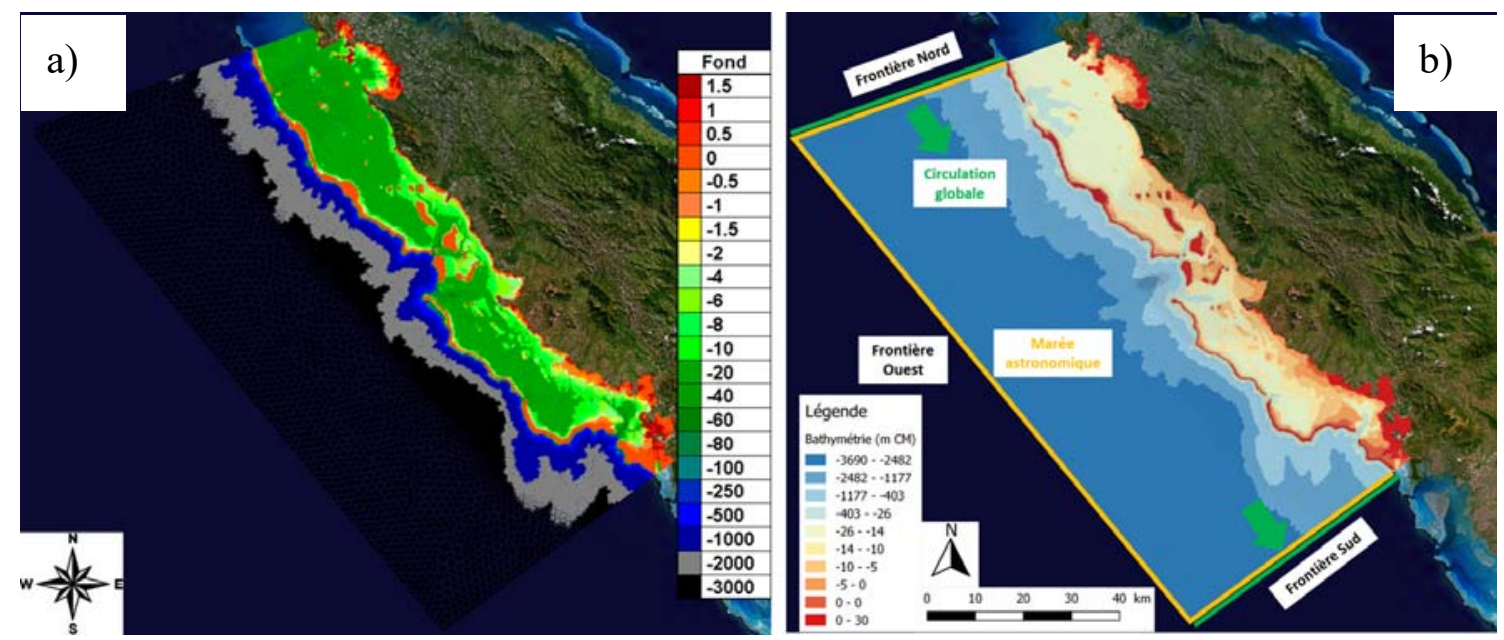

Fig. 3. a) Maillage et bathymétrie du modèle numérique ; b) Schéma des différents forçages.

La marée astronomique est forcée sur la frontière ouverte océanique grâce au modèle de prédiction de marée TPXO (EGBERT \& EROFEEVA, 2002).

Les circulations globales sont représentées dans le modèle grâce au développement d'un module spécifique permettant de forcer les vitesses en surface et à $1000 \mathrm{~m}$ de profondeur sur les frontières Nord et Sud à partir des données issues des modèles Mercator. Les vitesses sur les frontières pour les autres couches du maillage vertical sont ensuite interpolées en fonction de leur profondeur.

L'effet des vagues est pris en compte sur l'ensemble du domaine et sur toute la durée de la simulation. Le vent est imposé de manière constante sur l'emprise du modèle avec une série temporelle caractéristique du climat général.

La nature des terrains et les frottements qu'ils représentent sont pris en compte avec une loi de Manning. Le découpage des coefficients a été réalisé à partir des données du projet Koniambo (KBR, 2002).

\subsection{Calage}

Le modèle a été calé sur les niveaux d'eau à partir des prédictions du SHOM dans le port de Paagoumène (situé au Nord de la zone d'étude) et sur les vitesses à partir des données d'ADCP du projet Koniambo dans le passe de Duroc (Sud de la zone d'étude). Le modèle représente très bien les niveaux d'eau avec un biais inférieur à $2 \mathrm{~cm}$. Les données de courants sont également bien reproduites avec des indices statistiques satisfaisants (biais de l'ordre de $7 \mathrm{~cm} / \mathrm{s}$ ).

\section{Stratégie et résultats de modélisation}

\subsection{Hypothèses et stratégie de modélisation}

Concernant la modélisation des clapages, l'état de l'art (KBR, (2002); ALZIEU, 2000 ; BOUTIN, 2000) a permis de définir une représentation simpliste mais réaliste. 


\section{Thème 2 - Dynamique sédimentaire}

En effet, afin de représenter la remise en suspension des sédiments lors de la chute en "bloc" des matériaux clapés, les sédiments sont injectés dans la colonne d'eau à plusieurs profondeurs pour notamment prendre en compte le phénomène de changement de densité au niveau de la pycnocline. En fonction des vitesses de courant, 5\% en conditions calmes et $10 \%$ en conditions agitées des sédiments de la barge sont injectés en surface et au niveau de la pycnocline (à une profondeur de $60 \mathrm{~m}$ ) à chaque clapage. La chute en "bloc" du reste des sédiments avec une vitesse plus rapide n'est pas représentée dans le modèle.

Concernant le seuil d'impact des récifs, il a été défini au regard de la bibliographie (NSR, 2002; ERFTEMEIJER, 2012). Ainsi, les récifs sont considérés comme "impactés" lorsque les concentrations en matières en suspension seront supérieures à 10 $\mathrm{mg} / \mathrm{l}$ au niveau des profondeurs inférieures à $100 \mathrm{~m}$.

Deux sites potentiels de clapage situés à une distance supérieure à 3,5 km par rapport à l'isobathe $100 \mathrm{~m}$ et pour des profondeurs supérieures à $1000 \mathrm{~m}$. Etant donné ces contraintes, deux sites ont été définis à $4,5 \mathrm{~km}$ et à $6,5 \mathrm{~km}$ du récif. Les fréquences de clapage ont été calculées en fonction de la distance du site d'immersion et pour des conditions de travail usuelles.

Trois scénarios caractéristiques du climat général rencontré sur ce secteur de la Nouvelle Calédonie ont été modélisés sur une durée de 15 jours :

- Climat calme : vent offshore faible $(1-5 \mathrm{~m} / \mathrm{s})$, houle de Sud de hauteur 0,5m et courant océanique dirigé vers le Sud-Est avec des vitesses de l'ordre de 0,2 m/s,

- Saison fraiche : vent alternant entre alizés et coup d'Ouest (série temporelle issue de la station Duroc de Météo France), houle de Sud de hauteur 1,8 m et courant océanique nul,

- Saison des cyclones : régime d'alizés caractéristique de la saison avec des vents fort de $10 \mathrm{~m} / \mathrm{s}$ de Sud-Est en journée et des vents plus faibles pendant la nuit, houle de Sud de hauteur $1,8 \mathrm{~m}$ et courant océanique fort dirigé vers le Sud-Est avec des vitesses de l'ordre de $0,4 \mathrm{~m} / \mathrm{s}$.

\subsection{Résultats du modèle}

Les simulations des différents scénarios ont permis de caractériser les courants autour de la zone d'immersion. Ces courants sont globalement faibles $(<0,3 \mathrm{~m} / \mathrm{s})$ et engendrent donc une propagation spatiale limitée des sédiments.

Les sédiments injectés dans la colonne d'eau lors des clapages restent globalement très proches de la zone de clapage. En effet, compte tenu des valeurs de courants, les sédiments ne se propagent guère et tombent dans la colonne d'eau (où les courants sont de plus en plus faibles).

Les concentrations maximales relevées par le modèle sont de l'ordre de 15 à $20 \mathrm{mg} / \mathrm{l}$ dans la zone de clapage et ne dépassent pas les $5 \mathrm{mg} / \mathrm{l}$ en dehors de la zone de clapage. 


\section{XVèmes Journées Nationales Génie Côtier - Génie Civil \\ La Rochelle, 29 au 31 mai 2018}

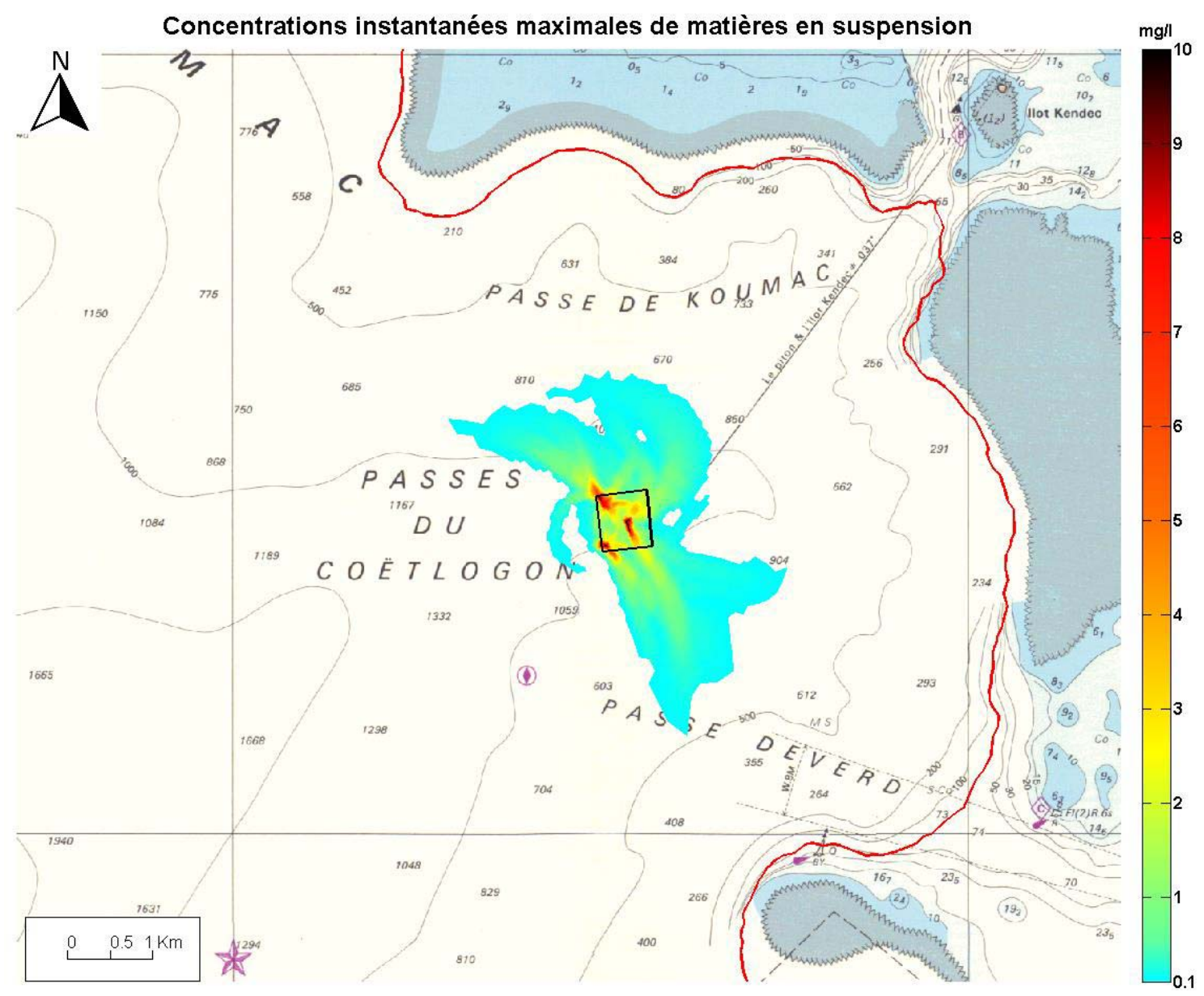

Figure 4. Emprise des concentrations instantanées maximales de MES pour le scénario 1 (conditions calmes).

L'influence des vents et de la houle est assez limitée mais leur rôle n'est pas pour autant négligeable d'où l'importance d'avoir caractérisé tous les forçages hydrodynamiques et de modéliser des scénarios caractéristiques des conditions océano-climatiques du site.

Les récifs ne sont pas impactés par les opérations de clapage quel que soit le scénario simulé. En effet, les concentrations en sédiments atteignant les récifs sont pour tous les scénarios très faibles.

Le scénario 3 (saison des cyclones) pour des courants océaniques globaux relativement forts semble être le scénario le plus défavorable avec les concentrations les plus fortes atteignant les profondeurs inférieures à $100 \mathrm{~m}$ de l'ordre de $0,13 \mathrm{mg} / 1$ contre $0,075 \mathrm{mg} / \mathrm{l}$ pour le scénario 1 (conditions calmes) et $0,02 \mathrm{mg} / 1$ pour le scénario 2 (saison fraîche). Ainsi les concentrations maximales observées au niveau des récifs sont donc environ 100 fois plus faibles que celles qui pourraient impacter les récifs. 


\section{Thème 2 - Dynamique sédimentaire}

\section{Conclusions}

Un modèle numérique hydrosédimentaire tridimensionnel a été mis place afin de représenter les différents processus hydrodynamiques et physiques mis en jeu (climat de houle, de vents, circulation océanique globale, ...) sur la façade Nord-Ouest de la Nouvelle-Calédonie.

Ce travail a permis d'étudier la dispersion des rejets par clapage pour différentes conditions océano-climatiques caractéristiques du climat général observé et différents secteurs. Les différents scénarios modélisés n'ont donné aucun dépassement du seuil d'impact des récifs.

Un deuxième site d'immersion plus au large a également été étudié entrainant une diminution de l'impact des clapages mais une augmentation des coûts (augmentation des temps de trajet et nécessité d'une barge supplémentaire pour garder la même cadence de travail).

De plus, des scénarios long-terme sur un mois de travail ont été effectués afin d'observer les effets cumulatifs des clapages successifs. Ce dernier travail a montré que le cumul des clapages n'a pas d'influence sur les impacts sur les récifs barrières. Finalement, la dispersion des matériaux clapés dépend principalement des conditions océanométéorologiques.

\section{Références bibliographiques}

ALZIEU C. (2000). Dragages et environnement marin. Etat des connaissances. Editions Ifremer, $225 \mathrm{p}$.

BOUTIN R. (2000). Dragage et rejets en mer. Les produits de type vase. Presses de l'ENPC, $307 \mathrm{p}$.

BRASSEUR P., BAHUREL P., BERTINO L., BIROL F., BRANKART J.-M., FERRY

N., LOSA S., REMY E., SCHRÖTER J., SKACHKO S., TESTUT C.E., TRANCHANT B., VAN LEEUWEN P.J., VERRON J. (2005). Data assimilation in operational ocean forecasting systems: The MERCATOR and MERSEA developments. Q. J. R. Met. Soc., Vol. 613, C, pp 3561-3582(22).

EGBERT G.D., EROFEEVA S.Y. (2002). Efficient inverse modeling of barotropic ocean tides. Journal of Atmospheric and Oceanic Technology, Vol. 19, pp 183-204. https://doi.org/10.1175/1520-0426(2002)019<0183:EIMOBO >2.0.CO;2

ERFTMEIJER P.L.A., RIEGL B., HOEKSEMA B.W., TODD P.T. (2012). Environmental impacts of dredging and other sediment disturbances on corals : A review. Marine Pollution Bulletin Vol. 64. pp 1737-1765.

KBR (2002). Koniambo Port project. Dredge spoil disposal report.

NSR. (2002). Koniambo Project, Physical Marine Investigations. NSR Environmental Consultants. 